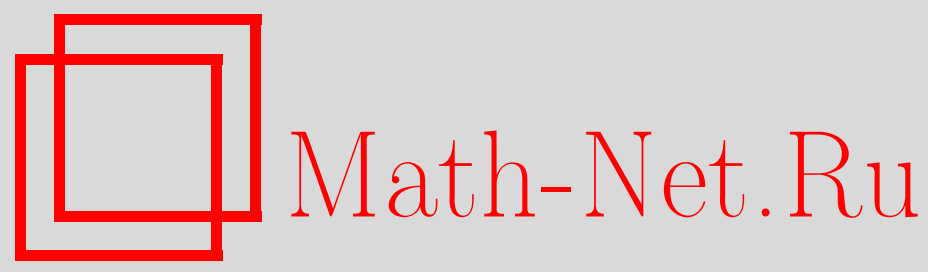

С. П. Горбиков, Топологическая эквивалентность одного типа локальных особенностей динамических систем с ударными взаимодействиями, Матем. заметки, 2001, том 70, выпуск 2, 181-194

DOI: https://doi.org/10.4213/mzm732

Использование Общероссийского математического портала Math-Net.Ru подразумевает, что вы прочитали и согласны с пользовательским соглашением http: //www . mathnet.ru/rus/agreement

Параметры загрузки:

IP : 54.92 .164 .108

26 апреля 2023 г., $17: 56: 49$

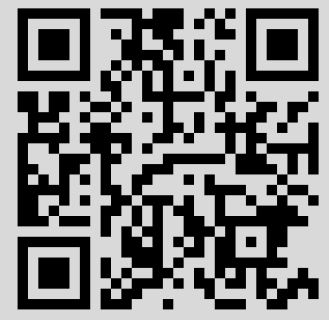




\title{
ТОПОЛОГИЧЕСКАЯ ЭКВИВАЛЕНТНОСТЬ ОДНОГО ТИПА ЛОКАЛЬНЫХ ОСОБЕННОСТЕЙ ДИНАМИЧЕСКИХ СИСТЕМ С УДАРНЫМИ ВЗАИМОДЕЙСТВИЯМИ \\ С. П. Горбиков
}

\begin{abstract}
Для выделенного типа локальных особенностей динамических систем с ударными взаимодействиями доказана теорема о топологической эквивалентности. Доказательство основано на установленном ранее результате об описании движений в окрестности выделенной локальной особенности с помощью гладких дифференциальных уравнений.

Библиограффия: 14 названий.
\end{abstract}

Введение. К настоящему времени качественная теория гладких динамических систем довольно хорошо развита (см. [1]-[3] и др.); для негладких динамических систем с непрерьвньм изменением переменных она успешно развивается (см. [4] и др.). Для динамических систем с ударными взаимодействиями изучались: локальная особенность одного вида для конкретной системы [5] и для неавтономной системы общего вида [6]; различные локальные особенности для динамических систем с ударными взаимодействиями общего вида [7]. В [8] для динамических систем с ударньми взаимодействиями классифицируются локальные особенности, для первых четырех выделенных типов локальных особенностей устанавливается топологическая эквивалентность соответствующих особенностей.

В данной работе рассматривается шестой [8] тип локальных особенностей, т.е. точка на гиперповерхности $S=0$ удара, в которой первая и вторая производная (в силу дифференциальных уравнений движения) уравнения этой гиперповерхности равна нулю, а третья производная отрицательна (движение системы происходит в области $S \geqslant 0$ ). Доказывается теорема о топологической эквивалентности малых окрестностей этих особенностей, причем используется полученное ранее [9] описание движений в малой окрестности (фазового пространства) локальной особенности шестого типа с помощью гладких дифференциальных уравнений.

1. Уравнения движения. В малой окрестности изучаемой здесь локальной особенности шестого типа [8] общие уравнения движения динамической системы с ударными взаимодействиями [7] удобнее представить в следующем виде [10]. Мгновенные ударные взаимодействия происходят на гиперповерхности $x_{n}=0$, по достижении которой фазовые переменные изменяются скачкообразно согласно формулам

$$
\begin{aligned}
& \bar{x}_{1}=H_{1}\left(x_{1}^{-}, \ldots, x_{n-1}^{-}\right)=x_{1}^{-} H_{11}\left(x_{1}^{-}, \ldots, x_{n-1}^{-}\right), \\
& \bar{x}_{i}=H_{i}\left(x_{1}^{-}, \ldots, x_{n-1}^{-}\right)=x_{i}^{-}+x_{1}^{-} H_{1 i}\left(x_{1}^{-}, \ldots, x_{n-1}^{-}\right), \quad i=2, \ldots, n-1,
\end{aligned}
$$


а при $x_{n}>0$ изменение фазовых переменных подчиняется дифференциальньм уравнениям вида

$$
\begin{aligned}
\dot{x}_{1} & =x_{1} \Phi_{11}\left(x_{1}, \ldots, x_{n}\right)+x_{2} \Phi_{12}\left(x_{1}, \ldots, x_{n}\right)+x_{n} \Phi_{1 n}\left(x_{1}, \ldots, x_{n}\right)=\Phi_{1}\left(x_{1}, \ldots, x_{n}\right), \\
\dot{x}_{i} & =\Phi_{i}\left(x_{1}, \ldots, x_{n}\right), \quad i=2, \ldots, n-1, \\
\dot{x}_{n} & =x_{1} \Phi_{n 1}\left(x_{1}, \ldots, x_{n}\right)+x_{n} \Phi_{n n}\left(x_{1}, \ldots, x_{n}\right)=\Phi_{n}\left(x_{1}, \ldots, x_{n}\right) .
\end{aligned}
$$

Фазовое пространство системы составляют точки $\left(x_{1}, \ldots, x_{n-1}, x_{n} \geqslant 0\right)$. В соотношениях (1) и (2) через $x_{1}^{-}, \ldots, x_{n-1}^{-}$и $x_{1}^{+}, \ldots, x_{n-1}^{+}$обозначены соответственно доударные и послеударные значения переменных; при этом $-1<H_{11}\left(0, x_{2}^{-}, \ldots, x_{n-1}^{-}\right)<0$; $\Phi_{n 1}\left(x_{1}, \ldots, x_{n-1}, 0\right)>0 ; \Phi_{12}\left(0, x_{2}, \ldots, x_{n-1}, 0\right)>0 ; \Phi_{2}(0, \ldots, 0)<0$. Функции $\Phi_{i}$, $i=2, \ldots, n-1, \Phi_{11}, \Phi_{12}, \Phi_{1 n}, \Phi_{n 1}, \Phi_{n n}$ и функции $H_{1 j}, j=1, \ldots, n-1$, определены и являются гладкими класса $C^{m}, m \geqslant 5$, в малых окрестностях точек $\left(x_{1}, \ldots, x_{n-1}, x_{n} \geqslant 0\right)$ и $\left(x_{1}^{-} \leqslant 0, x_{2}^{-}, \ldots, x_{n-1}^{-}\right)$соответственно.

На гиперповерхности удара согласно (2) выполнено равенство $\dot{x}_{n}=x_{1} \Phi_{n 1}\left(x_{1}, \ldots\right.$, $\left.x_{n-1}, 0\right)$. Поэтому [11, с. 171] фазовые траектории системы $(2)$ при $x_{1}=0$ касаются гиперховерхности $x_{n}=0$; при возрастании времени $t$ они выходят из точек $\left(x_{1}>0, x_{2}, \ldots\right.$, $\left.x_{n-1}, x_{n}=0\right)$, а при уменьшении $t-$ из точек $\left(x_{1}<0, x_{2}, \ldots, x_{n-1}, x_{n}=0\right)$. В силу (2) в точках многообразия $x_{1}=0, x_{n}=0$ при $x_{2}>0$ вьполняются соотношения

$$
\dot{x}_{n}=0, \quad \ddot{x}_{n}=x_{2} \Phi_{2}\left(0, x_{2}, \ldots, x_{n-1}, 0\right) \Phi_{n 1}\left(0, x_{2}, \ldots, x_{n-1}, 0\right)>0
$$

при $x_{2}<0$ - соотношения $\dot{x}_{n}=0, \ddot{x}_{n}<0 ;$ а в точке $M^{*}=(0, \ldots, 0)$ - соотношения

$$
\dot{x}_{n}=0, \quad \ddot{x}_{n}=0, \quad x_{n}^{\prime \prime \prime}=\frac{d^{3} x_{n}}{d t^{3}}=\Phi_{2}(0, \ldots, 0) \Phi_{12}(0, \ldots, 0) \Phi_{n 1}(0, \ldots, 0)<0
$$

K такому виду приводятся, например, механические системы с одной ударной парой с прямьм ударом, подчиняющимся гипотезе Ньютона, в окрестности локальной особенности шестого типа, характеризуемой условиями

$$
x_{n}=0, \quad \dot{x}_{n}=0, \quad \ddot{x}_{n}=0, \quad x_{n}^{\prime \prime \prime}=\frac{d^{3} x_{n}}{d t^{3}}<0,
$$

где производные берутся в силу дифференциальных уравнений движения системы, а $x_{n}$ обозначает расстояние между соударяющимися телами.

2. Особенности качественной структуры фазового пространства. В малой окрестности (см. рис. 1 ниже) точки $M^{*}(0, \ldots, 0)$, локальной особенности вида $(3)$, на многообразии $x_{n}=0, x_{1} \geqslant 0$ определено [10] точечное отображение $T=T_{2} T_{1}$ многообразия $x_{n}=0, x_{1} \geqslant 0$ в это же многообразие, где $T_{1}$ - отображение, переводящее точку $\left(x_{1} \geqslant 0, x_{2}, \ldots, x_{n-1}, 0\right)$ в точку $\left(x_{1}^{-} \leqslant 0, x_{2}^{-}, \ldots, x_{n-1}^{-}, 0\right)$, осуществляемое траекториями системы $(2)$, a $T_{2}$ - отображение, задаваемое формулами (1) ударных взаимодействий. 
Отображение $T$ задается [10] в малой окрестности начала координат формулами

$$
\begin{aligned}
\bar{x}_{1}= & {\left[x_{1}+t\left(x_{2}\left(a_{12}+\cdots\right)+x_{1}\left(a_{11}+\cdots\right)\right)+t^{2}\left(0,5 a_{2} a_{12}+\cdots\right)+t^{3} A_{1}\right]\left(b_{1}+\cdots\right) } \\
= & g_{1}\left(x_{1}, \ldots, x_{n-1}, t\right) \\
\bar{x}_{i}= & x_{i}+t\left(a_{i}+\cdots\right)+A_{i} t^{2}+\left[x_{1}+t\left(x_{2}\left(a_{12}+\cdots\right)+x_{1}\left(a_{11}+\cdots\right)\right)\right. \\
& \left.+t^{2}\left(0,5 a_{2} a_{12}+\cdots\right)+t^{3} A_{1}\right]\left(b_{i}+\cdots\right) \\
= & g_{i}\left(x_{1}, \ldots, x_{n-1}, t\right), \quad i=2, \ldots, n-1
\end{aligned}
$$

где $t$ - первый больший нуля корень уравнения

$$
\begin{aligned}
g_{n}\left(x_{1}, \ldots, x_{n-1}, t\right)= & x_{1}(1+\cdots)+t\left[x_{1}(\tilde{a}+\cdots)+x_{2}\left(\frac{a_{12}}{2}+\cdots\right)\right] \\
& +t^{2}\left(\frac{a_{12} a_{2}}{6}+\cdots\right)+t^{3} A_{n}=0
\end{aligned}
$$

Здесь и далее многоточие в аналитических выражениях обозначает члены более высокого порядка малости по $x_{i}, i=1, \ldots, n-1$, относительно рядом стоящих членов; $a_{i}=\Phi_{i}(0, \ldots, 0), a_{i j}=\Phi_{i j}(0, \ldots, 0), b_{i}=H_{1 i}(0, \ldots, 0), i, j=1, \ldots, n ; A_{j}, j=1, \ldots, n,-$ некоторые ограниченные функции; $\tilde{a}$ - некоторое число; $g_{j} \in C^{m}, j=1, \ldots, n$. Уравнение (5) при малых $x_{i}, i=1, \ldots, n-1$, и $x_{1} \geqslant 0$ имеет [10] требуемое решение.

На многообразии $x_{n}=0, x_{1} \geqslant 0$ существует в силу (4) и (5) следующее [10] множество $\nu_{1}$, являющееся образом многообразия $x_{1}=0, x_{n}=0, x_{2} \geqslant 0$ при действии отображения $T$ :

$$
\begin{gathered}
x_{1}=x_{2}^{2}\left(-\frac{3 a_{12}}{8 a_{2}} R+\cdots\right)=x_{2}^{2} \tilde{\nu}_{1}\left(x_{2}, \ldots, x_{n-1}\right)=\nu_{1}\left(x_{2}, \ldots, x_{n-1}\right), \\
\tilde{\nu}_{1} \in C^{m-2}, \quad x_{2} \leqslant 0, \quad R=-b_{1}=-H_{11}(0, \ldots, 0) .
\end{gathered}
$$

\section{Справедлива}

Теорема 1 [10]. Существуют такие достаточно малые числа $r$ и $r^{0}\left(r, r^{0}>0\right)$, что из каждой точки множества $G_{r}$, задаваемого соотношениями

$$
\sum_{i=1}^{n-1}\left|x_{i}\right| \leqslant r, \quad x_{n}=0, \quad x_{1}>0 \text { или } x_{1}=0, x_{2}>0
$$

выходят траектории бесконечноударных движений системы (1), (2), которые оканчиваются в точках многообразия

$$
x_{n}=0, \quad x_{1}=0, \quad x_{2}<0, \quad \sum_{i=2}^{n-1}\left|x_{i}\right| \leqslant r^{0} .
$$


ЗАМЕчАНИЕ. Под бесконечноударным движением понимается движение с бесконечньм числом ударных взаимодействий за конечное время [12], [13].

Бесконечноударные движения, о которых говорится в теореме 1 , допускают описание с помощью гладких дифференциальных уравнений. Для этого в множестве, определяемом условиями (7), следует произвести замену координат

$$
x_{1}=y_{1} y_{2}^{2}, \quad x_{i}=y_{i}, \quad i=2, \ldots, n-1 .
$$

Пусть к тому же $E^{r}$ - множество точек, координаты которых удовлетворяют условиЯM

$$
\sum_{i=2}^{n-1}\left|x_{i}\right| \leqslant r, \quad x_{n}=0, \quad 0 \leqslant x_{1} \leqslant \nu_{1}\left(x_{2}, \ldots, x_{n-1}\right), \quad x_{2} \leqslant 0,
$$

где функция $\nu_{1}$ определяется соотношениями (6). Тогда справедлива

Tеорема 2. Существует величина $r_{2}^{*}>0$ такая, что для любой точки $M$ множества $E_{2}^{r_{2}^{*}}$, задаваемого условиями (9) при $r=r_{2}^{*}$, имеет место следующее утверждение: все точки $M_{j}=T^{j}(M), j=1,2,3, \ldots$, лежст на проходящей через $M$ интегральной кривой системы дифференциальных уравнений

$$
\frac{d y_{i}}{d y_{1}}=f_{i}\left(y_{1}, \ldots, y_{n-1}\right)=y_{2} \tilde{f}_{i}, \quad i=2, \ldots, n-1
$$

əде $f_{i}=y_{2} \tilde{f}_{i} \in C^{m-4}$.

\section{3. Основная теорема.}

ТЕорема 3. Для любых двух систем вида (1), (2) (при одном и том же значении п) локальные особенности $M^{*}$ шестого типа, т.е. определяемые условиями (3), имеют достаточно малые окрестности в фазовом пространстве соответствующих систем, которые обладают одинаковой топологической структуpoü.

ДокАЗАТЕЛЬСТво. Пусть наряду с системой $(1),(2)$ задана система того же вида, т.е. определяемая при $z_{n}=0$ соотношениями

$$
\begin{aligned}
\bar{z}_{1} & =z_{1}^{-} \widehat{H}_{11}\left(z_{1}^{-}, \ldots, z_{n-1}^{-}\right)=\widehat{H}_{1} \\
\bar{z}_{i} & =z_{i}^{-}+z_{1}^{-} \widehat{H}_{1 i}\left(z_{1}^{-}, \ldots, z_{n-1}^{-}\right)=\widehat{H}_{i}, \quad i=2, \ldots, n-1,
\end{aligned}
$$

и при $z_{n}>0$ уравнениями

$$
\begin{aligned}
\dot{z}_{1} & =z_{1} \widehat{\Phi}_{11}\left(z_{1}, \ldots, z_{n}\right)+z_{2} \widehat{\Phi}_{12}\left(z_{1}, \ldots, z_{n}\right)+z_{n} \widehat{\Phi}_{1 n}\left(z_{1}, \ldots, z_{n}\right)=\widehat{\Phi}_{1} \\
z_{i} & =\widehat{\Phi}_{i}\left(z_{1}, \ldots, z_{n}\right), \quad i=2, \ldots, n-1, \\
\dot{z}_{n} & =z_{1} \widehat{\Phi}_{n 1}\left(z_{1}, \ldots, z_{n}\right)+z_{n} \widehat{\Phi}_{n n}\left(z_{1}, \ldots, z_{n}\right)=\widehat{\Phi}_{n}
\end{aligned}
$$

где соответствующие функции удовлетворяют условиям п. 1.

Пусть $M^{*}=\left(x_{1}^{*}=0, x_{2}^{*}, \ldots, x_{n-1}^{*}, x_{n}^{*}=0\right)$ и $N^{*}=\left(z_{1}^{*}=0, z_{2}^{*}, \ldots, z_{n-1}^{*}, z_{n}^{*}=0\right)$ любые две точки, удовлетворяющие условиям теоремы 3 соответственно для систем (1), 
(2) и (11), (12). После соответствующей замены переменных $u_{i}=x_{i}-x_{i}^{*}, i=1, \ldots, n$, и $w_{i}=z_{i}-z_{i}^{*}, i=1, \ldots, n$, проведенной для каждой из рассматриваемых систем, точки $M^{*}$ и $N^{*}$ перейдут в начала координат новых, выбранных систем координат, а условия вида (3) по-прежнему будут вьполняться. Далее для новых координат $u_{i}$ и $w_{i}$ сохраняются старые обозначения соответственно $x_{i}$ и $y_{i}, i=1, \ldots, n$.

Пусть теперь выбраны такие числа $r_{2}^{*}$ и $r_{2, z}^{*}$, при которых справедлива теорема 2 соответственно для точек $M^{*}$ и $N^{*}$ обеих рассматриваемых систем. Тогда для любой точки $M$ множества $E^{r_{2}^{*}}$ вида (9) (соответственно точки $N$ множества $E^{r_{2, z}^{*}}$ надлежащего вида) бесконечноударные движения, выходящие из точки $M$ (соответственно точки $N$ ) системы $(1),(2)$ (соответственно системы $(11),(12))$, описьваются следуюшими дифференциальными уравнениями.

Пусть для системы (1), (2) это будут уравнения (10), а для системы $(11),(12)$ - уравнения

$$
\begin{gathered}
\frac{d v_{i}}{d v_{1}}=\hat{f}_{i}\left(v_{1}, \ldots, v_{n-1}\right)=v_{2} h_{i}, \quad \hat{f}_{i} \in C^{m-4}, \quad i=2, \ldots, n-1, \\
z_{1}=v_{1} v_{2}^{2}, \quad z_{j}=v_{j}, \quad j=2, \ldots, n-1 .
\end{gathered}
$$

Пусть в новых координатах (8) через $E_{0}$ обозначается следующее множество на гиперповерхности удара:

$$
\sum_{i=2}^{n-1}\left|y_{i}\right| \leqslant r_{2}^{*}, \quad y_{2} \leqslant 0, \quad y_{1}=\tilde{\nu}_{1}\left(y_{2}, \ldots, y_{n-1}\right),
$$

где функция $\tilde{\nu}_{1}$ определяется в $(6)$, а через $\widetilde{E}_{0}$ обозначается соответствующее множество (задаваемое величиной $r_{2, z}^{*}$ ) для системы $(11),(12)$ в координатах $v_{1}, \ldots, v_{n-1}$ из (14).

Пусть теперь через $D\left(M^{*}\right)$ (соответственно $D\left(N^{*}\right)$ ) обозначается замкнутоемножество, которое образовано участками траекторий дифференциальных уравнений (10) (соответственно (13)), выходящими при уменьшении $y_{1}$ (соответственно $v_{1}$ ) из точек множества $E_{0}$ (соответственно $\left.\widetilde{E}_{0}\right)$ и продолжающиеся до пересечения с множеством $y_{1}=0$ (соответственно $v_{1}=0$ ). Такое пересечение существует и единственно в силу вида правых частей дифференциальных уравнений $(10),(13)$ и теорем о единственности и непрерьвной зависимости от начальных условий решений дифференциальных уравнений.

Пусть $T, T_{1}, T_{2}$ - отображения, определенные вьше для системы $(1),(2)$, а $\widetilde{T}_{1} \widetilde{T}_{1}, \widetilde{T}_{2}-$ отображения, определяемые так же, как и $T, T_{1}, T_{2}$, но для системы (11), (12). Тогда можно считать, что числа $r_{2}^{*}$ и $r_{2, z}^{*}$ взяты настолько малыми, что точки образа $T\left(E_{0}\right)$ (соответственно $\widetilde{T}\left(\widetilde{E}_{0}\right)$ ) множества $E_{0}$ (соответственно $\left.\widetilde{E}_{0}\right)$ при отображении $T$ (соответственно $\widetilde{T}$ ), лежащие на траекториях дифференциальных уравнений (10) (соответственно (13)), выходящих при уменьшении $y_{1}$ (уменьшении $v_{1}$ ) из соответствующих точек множества $E_{0}$ (соответственно $\left.\widetilde{E}_{0}\right)$, принадлежат разности множеств $D\left(M^{*}\right) \backslash E_{0}$ (соответственно $\left.D\left(N^{*}\right) \backslash \widetilde{E}_{0}\right)$, поскольку выполнены следующие условия:

1) образ $T\left(\nu_{1}\right)$ множества $\nu_{1}$, задаваемого соотношениями (6), при отображении $T$ (при уменьшении в случае необходимости величины $r_{2}^{*}$ ) имеет [10] вид $y_{1}=$ $\tilde{\nu}_{2}\left(y_{2}, \ldots, y_{n-1}\right)=\nu_{2}^{0}+\cdots$, где $\tilde{\nu}_{2} \in C^{m-2}$

2) имеет [10] место соотношение $\nu_{2}^{0}<-3 a_{12} R /\left(8 a_{2}\right)$, а поэтому можно считать (при уменьшении в случае необходимости величины $\left.r_{2}^{*}\right)$, что $\tilde{\nu}_{2}<\tilde{\nu}_{1}$ для всех рассматриваемых значений $y_{2}, \ldots, y_{n-1}$ при $y_{2}<0$. 
Пусть $D_{1}^{0}\left(M^{*}\right)=D_{1}^{0}$ (соответственно $\left.D_{2}^{0}\left(N^{*}\right)=D_{2}^{0}\right)$ обозначает замкнутое множество, которое заполнено траекториями дифференциальных уравнений (10) (соответственно $(13))$, заключенньми между множествами $T\left(E_{0}\right)\left(\right.$ соответственно $\left.\widetilde{T}\left(E_{0}\right)\right)$ и $E_{0}$ (соответственно $\widetilde{E}_{0}$ ). Тогда можно следуюшим образом установить топологическое соответствие $G$ между $D_{1}^{0}\left(M^{*}\right)$ и $D_{2}^{0}\left(N^{*}\right)$, при котором точки

$$
\begin{aligned}
& M^{0}=\left(y_{1}^{0}=-\frac{3 a_{12} R}{8 a_{2}}, y_{2}^{0}=0, y_{3}^{0}=0, \ldots, y_{n-1}^{0}=0\right), \\
& N^{0}=\left(v_{1}^{0}=-\frac{3 \hat{a}_{12} \widehat{R}}{8 \hat{a}_{2}}, v_{2}^{0}=0, v_{3}^{0}=0, \ldots, v_{n-1}^{0}=0\right)
\end{aligned}
$$

соответствуют одна другой (здесь и далее $\hat{a}_{2}=\widehat{\Phi}_{2}(0, \ldots, 0), \hat{a}_{12}=\widehat{\Phi}_{12}(0, \ldots, 0), 0<$ $\left.\widehat{R}=-\widehat{H}_{11}(0, \ldots, 0)<1\right)$ :

1) пусть $G$ - топологическое отображение множества $E_{0}$ на $\widetilde{E}_{0}$, задаваемое следующими соотношениями между картами многообразий $E_{0}$ и $\widetilde{E}_{0}$ :

$$
v_{i}=\frac{r_{2, z}^{*}}{r_{2}^{*}} y_{i}, \quad i=2, \ldots, n-1
$$

2) пусть $\varphi\left(y_{1}, y_{1}^{0}, M\right)$ и $\psi\left(v_{1}, v_{1}^{0}, N\right)$ - общее решение систем (10) с начальным условием $M=\varphi\left(y_{1}=y_{1}^{0}, y_{1}^{0}, M\right)$ и $(13)$ с начальньг условием $N=\psi\left(v_{1}=v_{1}^{0}, v_{1}^{0}, N\right)$ соответственно;

3) отображение $G$ на всем множестве $D_{1}^{0}\left(M^{*}\right)$ определяется как

$$
G\left(\varphi\left(y_{1}, y_{1}^{0}(M), M\right)\right)=\psi\left(v_{1}^{0}(N)-\left(y_{1}^{0}(M)-y_{1}\right) \frac{v_{1}^{0}(N)-\tilde{v}_{1}(N)}{y_{1}^{0}(M)-\tilde{y}_{1}(M)}, v_{1}^{0}(N), N\right)
$$

где

$$
\begin{gathered}
0<\tilde{y}_{1}(M) \leqslant y_{1} \leqslant y_{1}^{0}=y_{1}^{0}(M), \quad 0<\tilde{v}_{1}(N) \leqslant v_{1} \leqslant v_{1}^{0}=v_{1}^{0}(N), \\
N=\left(v_{1}^{0}(N), \ldots, v_{n-1}^{0}(N)\right)=G(M), \quad M=\left(y_{1}^{0}(M), \ldots, y_{n-1}^{0}(M)\right) \in E_{0}, \quad N \in \widetilde{E}_{0} ; \\
\varphi\left(\tilde{y}_{1}(M), y_{1}^{0}(M), M\right) \in T\left(E_{0}\right), \quad \psi\left(\tilde{v}_{1}(N), v_{1}^{0}(N), N\right) \in \tilde{T}\left(\tilde{E}_{0}\right) .
\end{gathered}
$$

Отображение $G$ является гомеоморфизмом в силу теорем о единственности и о непрерьвной зависимости от начальных условий решения системы дифференциальных уравнений.

Можно теперь следующим образом ввести на множестве $D\left(M^{*}\right)$ отображение $G_{T}$. Для любой точки $M \in D\left(M^{*}\right) \cap\left\{\left(y_{1}, \ldots, y_{n-1}\right) \mid y_{1}>0\right\}$

$$
G_{T}(M)=\widetilde{T}^{p} G T^{-p}(M)
$$

где целое число $p(p \geqslant 0)$ такое, что $T^{-p}(M) \in D_{1}^{0} \backslash T\left(E_{0}\right)$.

Для любой точки $M \in D\left(M^{*}\right) \cap\left\{\left(y_{1}, \ldots, y_{n-1}\right) \mid y_{1}=0\right\}$

$$
G_{T}(M)=\lim _{k \rightarrow+\infty} \widetilde{T}^{k} G\left(M_{\infty}\right)
$$

где точка $M_{\infty} \in E_{0}, \lim _{p \rightarrow+\infty} T^{p}\left(M_{\infty}\right)=M$, точка $N_{\infty}=G\left(M_{\infty}\right) \in \widetilde{E}_{0}$, а точка $M_{\infty}$ и $\lim _{k \rightarrow+\infty} \widetilde{T}^{k}\left(N_{\infty}\right)$ существуют и единственны по построению областей $D\left(M^{*}\right)$ и $D\left(N^{*}\right)$ в силу определения (15) множества $E_{0}$ и в силу справедливости дифференциальных уравнений (10) и (13). 
УТВЕРЖДЕНИЕ. Отображение $G_{T}$, определяемое формулами (17) и (18), является топологическим, переводит $D\left(M^{*}\right)$ на $D\left(N^{*}\right)$, а траектории отображсения $T$ в траектории отображения $\widetilde{T}$.

В справедливости этого утверждения можно убедиться аналогично тому, как это было сделано в процессе доказательства теоремы 2 в [8] при изучении свойств 1-3 соответствующего отображения $G_{T}$.

Теперь от координат $y_{1}, \ldots, y_{n-1}$ и $v_{1}, \ldots, v_{n-1}$ можно перейти к старьм координатам $x_{1}, \ldots, x_{n-1}$ и $z_{1}, \ldots, z_{n-1}$. Пусть $D_{x}\left(M^{*}\right)$ (соответственно $\left.D_{z}\left(N^{*}\right)\right)$ обозначает в старых координатах множество, соответствующее множеству $D\left(M^{*}\right)\left(D\left(N^{*}\right)\right)$ при замене (8) (соответственно (14)). При этом при $x_{2}=0$ (соответственно $z_{2}=0$ ) в точках множества $D_{x}\left(M^{*}\right)$ (соответственно $D_{z}\left(N^{*}\right)$ ) имеет место равенство $x_{1}=0$ (соответственно $\left.z_{1}=0\right)$. Пусть $G_{T, x}$ обозначает отображение $G_{T}$, но в старых координатах. Отображение $G_{T, x}$ переводит множество $D_{x}\left(M^{*}\right)$ на множество $D_{z}\left(N^{*}\right)$.

Замена (8) (и (14)) взаимно однозначна и взаимно непрерывна при $y_{2}=x_{2}<0$ (соответственно $v_{2}=z_{2}<0$ ), а множество точек $\left(0 \leqslant y_{1}, y_{2}=0, y_{3}^{M}, \ldots, y_{n-1}^{M}\right)$ (соответственно точек $\left.\left(0 \leqslant v_{1}, v_{2}=0, v_{3}^{N}, \ldots, v_{n-1}^{N}\right)\right)$ при этой замене переходит в одну точку $\left(x_{1}=0, x_{2}=0, x_{3}=y_{3}^{M}, \ldots, x_{n-1}=y_{n-1}^{M}\right)$ (соответственно в точку $\left.\left(z_{1}=0, z_{2}=0, z_{3}=v_{3}^{N}, \ldots, z_{n-1}=v_{n-1}^{N}\right)\right)$. Поэтому отображение $G_{T, x}$ на множестве $D_{x}\left(M^{*}\right)$ при $x_{2}<0$ является взаимно однозначньм и взаимно непрерывным.

Точке $M=\left(x_{1}=0, x_{2}=0, x_{3}^{M}, \ldots, x_{n-1}^{M}\right)$ множества $D_{x}\left(M^{*}\right)$ соответствует при отображении $G_{T, x}$ единственная точка

$$
G_{T, x}(M)=N=\left(z_{1}=0, z_{2}=0, z_{3}^{N}, \ldots, z_{n-1}^{N}\right) \in D_{z}\left(N^{*}\right)
$$

такая, что

$$
N_{v}=\left(v_{1}=0, v_{2}=0, v_{3}=z_{3}^{N}, \ldots, v_{n-1}=z_{n-1}^{N}\right)=G_{T}\left(M_{y}\right) \in D\left(N^{*}\right),
$$

где $M_{y}=\left(y_{1}=0, y_{2}=0, y_{3}=x_{3}^{M}, \ldots, y_{n-1}=x_{n-1}^{M}\right) \in D\left(M^{*}\right)$.

Действительно, при замене координат (8) (соответственно (14)) точке $M$ (точке $N$ ) соответствует в силу (10) траектория

$$
L=\left\{\left(y_{1}, \ldots, y_{n-1}\right) \mid y_{1}=\operatorname{var}, y_{2}\left(y_{1}\right) \equiv 0, y_{3}\left(y_{1}\right) \equiv x_{3}^{M}, \ldots, y_{n-1}\left(y_{1}\right) \equiv x_{n-1}^{M}\right\}
$$

дифференциальных уравнений (10) (соответственно траектория

$$
\left.S=\left\{\left(v_{1}, \ldots, v_{n-1}\right) \mid v_{1}=\operatorname{var}, v_{2}\left(v_{1}\right) \equiv 0, v_{2}\left(v_{1}\right) \equiv z_{3}^{N}, \ldots, v_{n-1}\left(v_{1}\right) \equiv z_{n-1}^{N}\right)\right\}
$$

дифференциальных уравнений $(13))$, причем $S=G_{T}(L)$ по построению отображения $G_{T}$.

Кроме того, отображение $G_{T, x}$ в точке

$$
M=\left(x_{1}=0, x_{2}=0, x_{3}^{M}, \ldots, x_{n-1}^{M}\right) \in D_{x}\left(M^{*}\right)
$$

является непрерывным.

Действительно, малой окрестности $\Omega_{x}(M) \subset D_{x}\left(M^{*}\right)$ (соответственно окрестности $\left.\widetilde{\Omega}_{z}\left(N^{*}\right) \subset D_{z}\left(N^{*}\right)\right)$ точки $M$ (точки $\left.N=G_{T, x}(M)\right)$ отвечает при замене (8) (замене (14)) малая окрестность определенной вьше траектории $L$ (траектории $S$ ), т.е. 
$\Omega(L) \subset D\left(M^{*}\right)$ (соответственно $\left.\widetilde{\Omega}(S) \subset D\left(N^{*}\right)\right)$, причем верно и обратное. В силу непрерьвной зависимости решений системы дифференциальных уравнений от начальных условий все точки малой окрестности $\Omega(L)$ (окрестности $\widetilde{\Omega}(S)$ ) лежат на траекториях дифференциальных уравнений (10) (соответственно (13)), которые проходят при $y_{1}=0\left(v_{1}=0\right)$ через точки малой окрестности $\Omega^{0}\left(M_{y}\right)\left(\right.$ соответственно $\left.\widetilde{\Omega}^{0}\left(N_{v}\right)\right)$ точки $M_{y}$ (точки $N_{v}$ ) на множестве $D\left(M^{*}\right) \cap\left\{\left(y_{1}, \ldots, y_{n}\right) \mid y_{1}=0\right\}$ (соответственно $\left.D\left(N^{*}\right) \cap\left\{\left(v_{1}, \ldots, v_{n}\right) \mid v_{1}=0\right\}\right)$, причем верно и обратное утверждение. В силу непрерьвности отображения $G_{T}$ найдется такая достаточно малая окрестность $\Omega^{0}\left(M_{y}\right)$, которая перейдет при отображении $G_{T}$ в любую наперед заданную окрестность $\widetilde{\Omega}^{0}\left(N_{v}\right)$. Поэтому всегда найдется такая достаточно малая окрестность $\Omega_{x}(M)$, которая перейдет при действии отображения $G_{T, x}$ в любую наперед заданную окрестность $\widetilde{\Omega}_{z}(N)$. Это доказьвает непрерьвность в точке $M\left(0,0, x_{3}^{M}, \ldots, x_{n-1}^{M}\right)$ отображения $G_{T, x}$.

Аналогично устанавливается и непрерывность обратного отображения $G_{T, x}^{-1}$ в точке $N\left(0,0, z_{3}^{N}, \ldots, z_{n-1}^{N}\right)$.

Таким образом, отображение $G_{T, x}$ является взаимно однозначньм и взаимно непрерьвньм, переводит множество $D_{x}\left(M^{*}\right)$ на множество $D_{z}\left(N^{*}\right)$, траектории отображения $T$ в траектории отображения $\widetilde{T}$. По построению отображения $G_{T}$ оно переводит точку

$$
M^{0}=\left(y_{1}^{0}=-\frac{3 a_{12} R}{8 a_{2}}, y_{2}^{0}=0, \ldots, y_{n-1}^{0}=0\right)
$$

в точку

$$
N^{0}=\left(v_{1}^{0}=-\frac{3 \hat{a}_{12} \widehat{R}}{8 \hat{a}_{2}}, v_{2}^{0}=0, \ldots, v_{n-1}^{0}=0\right),
$$

а, следовательно, $N^{*}=G_{T, x}\left(M^{*}\right)$.

Пусть $D_{1, x}^{0}$ (соответственно $D_{2, z}^{0}$ ) обозначает то множество, которому при замене (8) (при замене (14)) соответствует множество $D_{1}^{0}\left(M^{*}\right)$ (множество $D_{2}^{0}\left(N^{*}\right)$ ). Тогда можно предполагать, уменьшая при необходимости величину $r_{2}^{*}$ (соответственно $r_{2, z}^{*}$ ), что отображение $T$ (соответственно $\widetilde{T}$ ) является топологическим в точках множества $T^{-1}\left(D_{1, x}^{0}\right)$ (соответственно $\widetilde{T}^{-1}\left(D_{2, z}^{0}\right)$ ) потому, что при малых $x_{1}, \ldots, x_{n-1}$ отображение (1) имеет взаимно однозначное гладкое обратное отображение $T_{2}^{-1}$ по теореме о неявной функции, а отображение $T_{1}$ является взаимно однозначньм и взаимно непрерьвньп в силу теорем о единственности и о непрерывной зависимости от начальных условий решений систем дифференциальных уравнений.

Отображение $G_{T, x}$ можно теперь доопределить в точках множества $T^{-1}\left(D_{1, x}^{0}\right)$ :

$$
N=G_{T, x}(M)=\widetilde{T}^{-1} G_{T, x} T(M),
$$

где $M \in T^{-1}\left(D_{1, x}^{0}\right), N \in \widetilde{T}^{-1}\left(D_{2, z}^{0}\right)$. Отображение (19) является топологическим в точках множества $T^{-1}\left(D_{1, x}^{0}\right)$, так как таковыми являются его составляющие.

При этом (рис. 1$)$ множество $T^{-1}\left(D_{1, x}^{0}\right)$ заключается между множеством (6) и множеством $x_{1}=0, x_{2} \geqslant 0$ на многообразии $x_{n}=0, x_{1} \geqslant 0$, а множество $D_{x}\left(M^{*}\right)$ расположено между множествами $x_{1}=0, x_{2} \leqslant 0$ и $\nu_{1}$ на том же многообразии. Поэтому множество $D_{x}\left(M^{*}\right) \cup T^{-1}\left(D_{1, x}^{0}\right)\left(\right.$ соответственно $\left.D_{z}\left(N^{*}\right) \cup \widetilde{T}^{-1}\left(D_{2, z}^{0}\right)\right)$ образует окрестность точки $M^{*}$ (точки $\left.N^{*}\right)$ на многообразии $x_{n}=0, x_{1} \geqslant 0$ (соответственно $z_{n}=0$, $\left.z_{1} \geqslant 0\right)$, а точка $M^{*}$ (точка $\left.N^{*}\right)$ - внутренняя точка этой окрестности. 


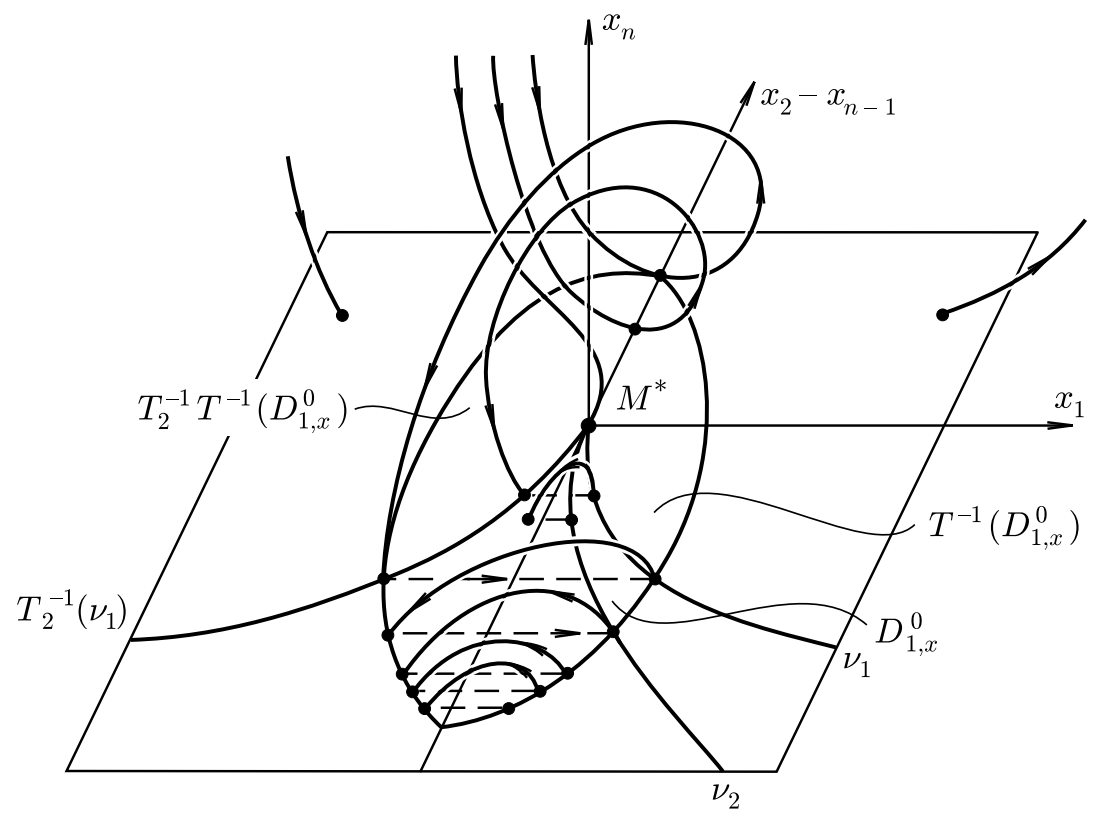

РИС. 1

Образ множества $D_{x}\left(M^{*}\right) \cup T^{-1}\left(D_{1, x}^{0}\right)$ при отображении $T_{1}$ есть множество, расположенное между множеством $T_{2}^{-1}\left(\nu_{1}\right)$ и множеством $x_{1}=0, x_{2} \leqslant 0$ на многообразии $x_{n}=0, x_{1} \leqslant 0$. А множество $T_{2}^{-1}\left(T^{-1}\left(D_{1, x}^{0}\right)\right)$ заключено между множеством $T_{2}^{-1}\left(\nu_{1}\right)$ и $x_{1}=0, x_{2} \geqslant 0$ на многообразии $x_{n}=0, x_{1} \leqslant 0$. Множество

$$
D_{x}\left(M^{*}\right) \cup T^{-1}\left(D_{1, x}^{0}\right) \cup T_{1}\left(D_{x}\left(M^{*}\right) \cup T^{-1}\left(D_{1, x}^{0}\right)\right) \cup T_{2}^{-1}\left(T^{-1}\left(D_{1, x}^{0}\right)\right)
$$

образует окрестность точки $M^{*}$ на многообразии $x_{n}=0$, а $M^{*}$ - его внутренняя точка. Аналогичное утверж дение справедливо и относительно точки $N^{*}$.

В точках множества $T_{2}^{-1}\left(T^{-1}\left(D_{1, x}^{0}\right)\right)$ отображение $G_{T, x}$ можно определить следующим образом:

$$
N=G_{T, x}(M)=\widetilde{T}_{2}^{-1} G_{T, x} T_{2}(M)
$$

где

$$
M \in T_{2}^{-1}\left(T^{-1}\left(D_{1, x}^{0}\right)\right), \quad N \in \widetilde{T}_{2}^{-1}\left(\widetilde{T}^{-1}\left(D_{2, z}^{0}\right)\right)
$$

Отображение (20) является топологическим в точках множества $T_{2}^{-1}\left(T^{-1}\left(D_{1, x}^{0}\right)\right)$, так как таковыми являются его составляющие.

Поэтому отображение $G_{T, x}$ определено и является топологическим на множестве

$$
D_{x}\left(M^{*}\right) \cup T^{-1}\left(D_{1, x}^{0}\right) \cup T_{2}^{-1}\left(T^{-1}\left(D_{1, x}^{0}\right)\right) ;
$$

оно переводит траектории отображения $T$ в траектории отображения $\widetilde{T}$, а точку $M^{*}-$ в точку $N^{*}$. 
Пусть теперь $E_{1}\left(M^{*}\right)$ (соответственно $\left.\widetilde{E}_{1}\left(N^{*}\right)\right)$ - множество, которое в фазовом пространстве системы (1), (2) (соответственно системы $(11),(12))$ составляют участки траекторий системы (2) (системы (12)), выходящие при увеличении времени из точек множества $D_{x}\left(M^{*}\right) \cup T^{-1}\left(D_{1, x}^{0}\right)$ (соответственно множества $\left.D_{z}\left(N^{*}\right) \cup \widetilde{T}^{-1}\left(D_{2, z}^{0}\right)\right)$ и пересекающие гиперповерхность удара $x_{n}=0$ (соответственно $\left.z_{n}=0\right)$.

Пусть, кроме того, $x_{i}=\varphi_{i}\left(t, x_{1}^{0}, x_{2}^{0}, \ldots, x_{n}^{0}\right), i=1, \ldots, n$ (соответственно $z_{i}=\psi_{i}(t$, $\left.\left.z_{1}^{0}, z_{2}^{0}, \ldots, z_{n}^{0}\right), i=1, \ldots, n\right)$, есть общее решение системы (2) (системы (12)) с начальными условиями $x_{i}^{0}=\varphi_{i}\left(0, x_{1}^{0}, \ldots, x_{n}^{0}\right), i=1, \ldots, n\left(\right.$ соответственно $z_{i}^{0}=\psi_{i}\left(0, z_{1}^{0}, \ldots, z_{n}^{0}\right)$, $i=1, \ldots, n)$.

Тогда можно задать топологическое отображение $G_{1}$ множества $E_{1}\left(M^{*}\right)$ на $\widetilde{E}_{1}\left(N^{*}\right)$ :

$$
G_{1}(M)=\left(\psi_{1}\left(|\tau| \frac{\tilde{\theta}}{\theta}, z_{1}^{N}, \ldots, z_{n-1}^{N}, 0\right), \ldots, \psi_{n}\left(|\tau| \frac{\tilde{\theta}}{\theta}, z_{1}^{N}, \ldots, z_{n-1}^{N}, 0\right)\right)
$$

где

$$
\begin{gathered}
M\left(x_{1}, \ldots, x_{n}\right) \in E_{1}\left(M^{*}\right), \quad\left(z_{1}^{N}, \ldots, z_{n-1}^{N}\right)=G_{T, x}\left(M_{-1}\right), \\
M_{-1}=\left(\varphi_{1}\left(\tau, x_{1}, \ldots, x_{n}\right), \ldots, \varphi_{n-1}\left(\tau, x_{1}, \ldots, x_{n}\right)\right)=\left(x_{-1}, x_{-2}, \ldots, x_{-(n-1)}\right),
\end{gathered}
$$

$\tau$ - ближайший к нулю неположительный корень уравнения $\varphi_{n}\left(t, x_{1}, \ldots, x_{n}\right)=0, \theta-$ ближайший к нулю положительный корень уравнения $\varphi_{n}\left(t, x_{-1}, \ldots, x_{-(n-1)}, 0\right)=0$, $\tilde{\theta}$ - ближайший к нулю положительньй корень уравнения $\psi_{n}\left(t, z_{1}^{N}, \ldots, z_{n-1}^{N}, 0\right)=0$. Считается, что

$$
G_{1}\left(\left(0, x_{2} \leqslant 0, x_{3}, \ldots, x_{n-1}, 0\right)\right)=\left(0, z_{2}, \ldots, z_{n-1}, 0\right),
$$

где

$$
\left(0, z_{2}, \ldots, z_{n-1}\right)=G_{T, x}\left(\left(0, x_{2} \leqslant 0, x_{3}, \ldots, x_{n-1}\right)\right) .
$$

Пусть $E_{2}\left(M^{*}\right)$ (соответственно $\widetilde{E}_{2}\left(N^{*}\right)$ ) - множество, которое в фазовом пространстве системы (1), (2) (соответственно системы (11), (12)) составляют следующие участки траекторий системы (2) (соответственно системы (12)), выходящие при уменьшении времени из точек множества $T_{2}^{-1}\left(T^{-1}\left(D_{1, x}^{0}\right)\right)$ (соответственно из точек множества $\left.\widetilde{T}_{2}^{-1}\left(\widetilde{T}^{-1}\left(D_{2, z}^{0}\right)\right)\right)$ :

$$
\begin{gathered}
x_{i}=\varphi_{i}\left(t, x_{1}^{0}, \ldots, x_{n-1}^{0}, x_{n}^{0}=0\right), \quad i=1, \ldots, n \\
\left(\text { соответственно } z_{i}=\psi_{i}\left(t, z_{1}^{0}, \ldots, z_{n-1}^{0}, z_{n}^{0}=0\right), i=1, \ldots, n\right),
\end{gathered}
$$

где $M^{0}=\left(x_{1}^{0}, \ldots, x_{n-1}^{0}\right) \in T_{2}^{-1}\left(T^{-1}\left(D_{1, x}^{0}\right)\right)\left(\right.$ соответственно $N^{0}=\left(z_{1}^{0}, \ldots, z_{n-1}^{0}\right) \in$ $\left.\widetilde{T}_{2}^{-1}\left(\widetilde{T}^{-1}\left(D_{2, z}^{0}\right)\right)\right), 0 \geqslant t \geqslant \tau+t_{2}\left(x_{2}^{0}, \ldots, x_{n-1}^{0}\right)$ (соответственно $0 \geqslant t \geqslant \hat{\tau}=\hat{\tau}\left(z_{1}^{0}, \ldots\right.$, $\left.z_{n-1}^{0}\right)$; функция $t_{2}\left(x_{2}^{0}, \ldots, x_{n-1}^{0}\right)$ имеет вид

$$
t_{2}\left(x_{2}^{0}, \ldots, x_{n-1}^{0}\right)= \begin{cases}0 & \text { при } x_{2}^{0} \geqslant 0, \\ \text { наибольший отрищательньй корень уравнения } & \\ \varphi_{n}\left(t, \gamma_{1}\left(x_{2}^{0}, \ldots, x_{n-1}^{0}\right), x_{2}^{0}, \ldots, x_{n-1}^{0}, 0\right)=0 & \text { при } x_{2}^{0}<0 .\end{cases}
$$


Здесь и далее используется функция, задающая множество $T_{2}^{-1}\left(\nu_{1}\right)$ (соответственно множество $\widetilde{T}_{2}^{-1}\left(\hat{\nu}_{1}\right)$, где множество $\hat{\nu}_{1}$ - образ при отображении $\widetilde{T}$ многообразия $z_{1}=0$, $\left.z_{n}=0, z_{2} \geqslant 0\right)$ следуюшими (в силу (1) и (6)) соотношениями:

$$
x_{1}=\gamma_{1}\left(x_{2}, \ldots, x_{n-1}\right)=x_{2}^{2}\left(\frac{3 a_{12}}{8 a_{2}}+\cdots\right), \quad x_{2} \leqslant 0, \quad \gamma_{1} \in C^{m-2}
$$

\section{(соответственно}

$$
\left.z_{1}=\hat{\gamma}_{1}\left(z_{2}, \ldots, z_{n-1}\right)=z_{2}^{2}\left(\frac{3 \hat{a}_{12}}{8 \hat{a}_{2}}+\cdots\right), \quad z_{2} \leqslant 0, \quad \hat{\gamma}_{1} \in C^{m-2}\right)
$$

Для определения функции $\hat{\tau}\left(z_{1}^{0}, \ldots, z_{n-1}^{0}\right)$ будет далее использована функция $k\left(x_{2}^{0}, \ldots\right.$, $\left.x_{n-1}^{0}\right)$, которая задается следующим образом:

1) если у точки $M^{0}$ координата $x_{2}^{0}<0$, то

$$
k=\frac{\tilde{\theta}}{\theta}
$$

где $\theta$ - ближайший к нулю отрицательный корень уравнения

$$
\varphi_{n}\left(t, \gamma_{1}\left(x_{2}^{0}, \ldots, x_{n-1}^{0}\right), x_{2}^{0}, \ldots, x_{n-1}^{0}, 0\right)=0
$$

$\tilde{\theta}$ - ближайший к нулю отрицательный корень уравнения

$$
\begin{gathered}
\psi_{n}\left(t, z_{-1}, z_{-2}, \ldots, z_{-(n-1)}, 0\right)=0 \\
\left(z_{-1}, z_{-2}, \ldots, z_{-(n-1)}\right)=N_{-1}=G_{T, x}\left(M_{0}^{0}\right) \in \widetilde{T}_{2}^{-1}\left(\hat{\nu}_{1}\right) \\
M_{0}^{0}=\left(\gamma_{1}\left(x_{2}^{0}, \ldots, x_{n-1}^{0}\right), x_{2}^{0}, \ldots, x_{n-1}^{0}\right)
\end{gathered}
$$

2 ) если у точки $M^{0}$ координата $x_{2}^{0}>0$, то также верно $(22)$, но уже $\theta$ - ближайший к нулю положительньй корень уравнения

$$
\varphi_{n}\left(t, 0, x_{2}^{0}, \ldots, x_{n-1}^{0}, 0\right)=0
$$

$\tilde{\theta}$ - ближайший к нулю положительньй корень уравнения

$$
\psi_{n}\left(t, 0, z_{-2}, \ldots, z_{-(n-1)}, 0\right)=0
$$

здесь

$$
\left(z_{-1}=0, z_{-2}, \ldots, z_{-(n-1)}\right)=N_{-1}=G_{T, x}\left(M_{0}^{1}\right), \quad M_{0}^{1}=\left(0, x_{2}^{0}, \ldots, x_{n-1}^{0}\right)
$$

3) если у точки $M^{0}$ координата $x_{2}^{0}=0$, то

$$
k\left(x_{2}^{0}=0, x_{3}^{0}, \ldots, x_{n-1}^{0}\right)=\lim _{x_{2} \rightarrow+0} k\left(x_{2}, x_{3}^{0}, \ldots, x_{n-1}^{0}\right)=\lim _{x_{2} \rightarrow-0} k\left(x_{2}, x_{3}^{0}, \ldots, x_{n-1}^{0}\right) .
$$


Здесь первьй предел существует и равен $\left(r_{2, z}^{*} / r_{2}^{*}\right)\left(a_{2} / \hat{a}_{2}\right)+\cdots$ в силу определения $G_{T, x}\left(M_{0}^{1}\right),(16),(14),(8)$ и того, что решение уравнения (5) для точек множества $x_{n}=0$, $x_{1}=0, x_{2} \geqslant 0$ имеет [10] вид $t=x_{2} \tilde{\tilde{t}}=x_{2}\left(-3 / a_{2}+\cdots\right), \tilde{\tilde{t}} \in C^{m-2}$, а второй предел существует и равен первому в силу определения функции $k$. Кроме того, при достаточно малых $r_{2}^{*}$ и $r_{2, z}^{*}$ имеет место неравенство $k\left(x_{2}^{0}, x_{3}^{0}, \ldots, x_{n-1}^{0}\right)>0$, а функция $k$ является непрерьвной.

Функция $\hat{\tau}\left(z_{1}^{0}, \ldots, z_{n-1}^{0}\right)$, введенная после $(21)$, имеет вид

$$
\hat{\tau}=k\left(x_{2}^{0}, \ldots, x_{n-1}^{0}\right)\left[\tau+t_{2}\left(x_{2}^{0}, \ldots, x_{n-1}^{0}\right)\right],
$$

где

$$
N^{0}\left(z_{1}^{0}, \ldots, z_{n-1}^{0}\right)=G_{T, x}\left(M^{0}\right), \quad M^{0}=\left(x_{1}^{0}, \ldots, x_{n-1}^{0}\right) \in T_{2}^{-1}\left(T^{-1}\left(D_{1, x}^{0}\right)\right)
$$

константа $\tau$ (соответственно функция $\left.\hat{\tau}=k\left(\tau+t_{2}\right)\right)$ - достаточно малая отрицательная величина (соответственно функция) такая, что для всех участков траекторий $(21)$ имеет место неравенство

$$
\varphi_{n}\left(t, x_{1}^{0}, \ldots, x_{n-1}^{0}, 0\right)>0 \quad\left(\text { соответственно } \psi_{n}\left(t, z_{1}^{0}, \ldots, z_{n-1}^{0}, 0\right)>0\right)
$$

при $M^{0} \notin T_{2}^{-1}\left(\nu_{1}\right)$ (соответственно при $\left.N^{0} \notin \widetilde{T}_{2}^{-1}\left(\hat{\nu}_{1}\right)\right)$ для $0>t \geqslant \tau+t_{0}($ соответственно для $0>t \geqslant \hat{\tau}$ ), а при $M^{0} \in T_{2}^{-1}\left(\nu_{1}\right)$ (соответственно при $N^{0} \in \widetilde{T}_{2}^{-1}\left(\hat{\nu}_{1}\right)$ ) для всех $0>t \geqslant \tau+t_{0}$ (соответственно для всех $0>t \geqslant \hat{\tau}$ ), за исключением такого $t$, при котором участок траектории (21) первьй раз попадает при уменьшении $t$ на гиперповерхность $x_{n}=0$ (соответственно на гиперповерхность $\left.z_{n}=0\right)$.

Существования таких $\tau$ и $\hat{\tau}$ можно добиться, уменьшая при необходимости величины $r_{2}^{*}$ и $r_{2, z}^{*}$ соответственно.

Множества $E_{1}\left(M^{*}\right)$ и $E_{2}\left(M^{*}\right)$ имеют общую часть, которая состоит из участков траекторий системы (2), выходящих при уменьшении времени $t$ из точек множества $T_{2}^{-1}\left(\nu_{1}\right)$ $\cap T_{2}^{-1}\left(T^{-1}\left(D_{1, x}^{0}\right)\right)$ и впервые пересекающих многообразие $x_{n}=0$ в точках многообразия $x_{1}=0, x_{2} \geqslant 0$ (последние принадлежат множеству $T^{-1}\left(D_{1, x}^{0}\right)$ ). Аналогичное утверждение справедливо и относительно множеств $\widetilde{E}_{1}\left(N^{*}\right)$ и $\widetilde{E}_{2}\left(N^{*}\right)$.

Множество $E_{1}\left(M^{*}\right) \cup E_{2}\left(M^{*}\right)$ (соответственно $\left.\widetilde{E}_{1}\left(N^{*}\right) \cup \widetilde{E}_{2}\left(N^{*}\right)\right)$ образует окрестность точки $M^{*}$ (точки $\left.N^{*}\right)$ в фазовом пространстве системы $(1),(2)($ системы $(11),(12)$, а точка $M^{*}$ (точка $\left.N^{*}\right)$ - его внутренняя точка.

Теперь можно задать отображение $G_{2}$ множества $E_{2}\left(M^{*}\right)$ на $\widetilde{E}_{2}\left(N^{*}\right)$ :

$$
\begin{aligned}
G_{2}(M)= & \left(\psi_{1}\left(-\tau_{M} k\left(x_{-2}, \ldots, x_{-(n-1)}\right), z_{1}^{M}, \ldots, z_{n-1}^{M}, 0\right), \ldots,\right. \\
& \left.\psi_{n}\left(-\tau_{M} k\left(x_{-2}, \ldots, x_{-(n-1)}\right), z_{1}^{M}, \ldots, z_{n-1}^{M}, 0\right)\right),
\end{aligned}
$$

где

$$
\begin{aligned}
& M\left(x_{1}, \ldots, x_{n}\right) \in E_{2}\left(M^{*}\right)\left(z_{1}^{M}, \ldots, z_{n-1}^{M}\right)=G_{T, x}\left(M_{-1}\right) \in \widetilde{T}_{2}^{-1}\left(\widetilde{T}^{-1}\left(D_{2, z}^{0}\right)\right), \\
& M_{-1}=\left(\varphi_{1}\left(\tau_{M}, x_{1}, \ldots \ldots, x_{n}\right), \ldots, \varphi_{n-1}\left(\tau_{M}, x_{1}, \ldots, x_{n}\right)\right)=\left(x_{-1}, \ldots, x_{-(n-1)}\right) \\
& \quad \in T_{2}^{-1}\left(T^{-1}\left(D_{1, x}^{0}\right)\right),
\end{aligned}
$$


$\tau_{M}$ - ближайший к нулю неотрицательный корень уравнения $\varphi_{n}\left(t, x_{1}, \ldots \ldots, x_{n}\right)=0$, если $M$ не принадлежит множеству $S_{0}$ фазовых траекторий системы $(2)$, выходящих при уменьшении времени $t$ из точек множества $T_{2}^{-1}\left(\nu_{1}\right)$, а если $M \in S_{0}$, то $\tau_{M}$ - неотрицательный корень уравнения $\varphi_{n}\left(t, x_{1}, \ldots, x_{n}\right)=0$, которьй соответствует попаданию фазовой точки на множество $T_{2}^{-1}\left(\nu_{1}\right)$; функция $k$ определена вьше.

Отображение $G_{2}$ является взаимно однозначным и непрерывным в силу своего определения и соответствующих теорем о решениях дифференциальных уравнений.

Обратное отображение $G_{2}^{-1}$ можно задать формулой вида (23) с очевидными изменениями:

$$
\begin{aligned}
G_{2}^{-1}(N)= & \left(\varphi_{1}\left(-\frac{\tau_{N}}{k\left(x_{2}^{N}, \ldots, x_{n-1}^{N}\right)}, x_{1}^{N}, \ldots, x_{n-1}^{N}, 0\right), \ldots,\right. \\
& \left.\varphi_{n}\left(-\frac{\tau_{N}}{k\left(x_{2}^{N}, \ldots, x_{n-1}^{N}\right)}, x_{1}^{N}, \ldots, x_{n-1}^{N}, 0\right)\right),
\end{aligned}
$$

где

$$
\begin{gathered}
N\left(z_{1}, \ldots, z_{n}\right) \in \widetilde{E}_{2}\left(N^{*}\right), \quad\left(x_{1}^{N}, \ldots, x_{n-1}^{N}\right)=G_{T, x}^{-1}\left(N_{-1}\right) \in T_{2}^{-1}\left(T^{-1}\left(D_{1, x}^{0}\right)\right), \\
N_{-1}=\left(\psi_{1}\left(\tau_{N}, z_{1}, \ldots, z_{n}\right), \ldots, \psi_{n-1}\left(\tau_{N}, z_{1}, \ldots, z_{n}\right)\right) \in \widetilde{T}_{2}^{-1}\left(\widetilde{T}^{-1}\left(D_{2, z}^{0}\right)\right),
\end{gathered}
$$

$\tau_{N}$ - ближайший к нулю неотрицательный корень уравнения $\psi_{n}\left(t, z_{1}, \ldots, z_{n}\right)=0$, если $N$ не принадлежит множеству $\widetilde{S}_{0}$ фазовьх траекторий системы $(12)$, выходящих при уменьшении времени из точек множества $\widetilde{T}_{2}^{-1}\left(\hat{\nu}_{1}\right)$, а если $N \in \widetilde{S}_{0}$, то $\tau_{N}$ - неотрицательный корень уравнения $\psi_{n}\left(t, z_{1}, \ldots, z_{n}\right)=0$, которьй соответствует попаданию фазовой точки на множество $\widetilde{T}_{2}^{-1}\left(\hat{\nu}_{1}\right)$. Поскольку, как указьвалось вьше, $k>0$, то отображение $(24)$ является непрерывным, а отображение $G_{2}$ - топологическим.

Кроме того, отображение $G_{2}$ переводит траектории системы $(2)$ в траектории системы (12), а точку $M^{*}$ в точку $N^{*}$.

Теперь можно задать отображение $G$ множества $E_{1}\left(M^{*}\right) \cup E_{2}\left(M^{*}\right)$ на множество $\widetilde{E}_{1}\left(N^{*}\right) \cup \widetilde{E}_{2}\left(N^{*}\right)$ следующим образом:

$$
G(M)= \begin{cases}G_{1}(M), & \text { если } M \in E_{1}\left(M^{*}\right), \\ G_{2}(M), & \text { если } M \in E_{2}\left(M^{*}\right) .\end{cases}
$$

\section{Поскольку}

$1)$ в силу определения отображений $G_{1}$ и $G_{2}, G_{i}\left(E_{i}\left(M^{*}\right)\right)=\widetilde{E}_{i}\left(N^{*}\right), i=1,2$, отображения $G_{1}$ и $G_{2}$ на множестве $E_{1}\left(M^{*}\right) \cap E_{2}\left(M^{*}\right)$ совпадают и $G_{i}\left(E_{1}\left(M^{*}\right) \cap E_{2}\left(M^{*}\right)\right)=$ $\widetilde{E}_{1}\left(N^{*}\right) \cap \widetilde{E}_{2}\left(N^{*}\right), i=1,2$;

2) множества $E_{i}\left(M^{*}\right), \widetilde{E}_{i}\left(N^{*}\right), i=1,2$, являются замкнутьми;

3 ) отображения $G_{i}, i=1,2$, являются топологическими, то $[14$, с. 70$]$ отображение $G$ является топологическим, переводит множество $E_{1}\left(M^{*}\right) \cup$ $E_{2}\left(M^{*}\right)$ на множество $\widetilde{E}_{1}\left(M^{*}\right) \cup \widetilde{E}_{2}\left(N^{*}\right)$. Кроме того, в силу своего определения отображение $G$ переводит точку $M^{*}$ в точку $N^{*}$, а траектории системы $(1),(2)$ в траектории системы (11), (12). Теорема 3 доказана. 


\section{СПИСОК ЦИТИРОВАННОЙ ЛИТЕРАТУРЫ}

[1] Андронов А. А., Витт А. А., Хайкин С.Э. Теория колебаний. М.: Физматгиз, 1959.

[2] Немыцкий В. В., Степанов В. В. Качественная теория дифференциальных уравнений. 2-е изд. М.-Л.: ГИТТЛ, 1949.

[3] Андронов А. А., Леонтович Е. А., Гордон И. И., Майер А. Г. Качественная теория динамических систем второго порядка. М.: Наука, 1966.

[4] Филиппов А.Ф. Дифференциальные уравнения с разрьвной правой частью. М.: Наука, 1985.

[5] Денисов Г. Г., Неймарк Ю.И., Сандалов В. М., Цветков Ю. В. Об обкате ротора по жесткому подшипнику // Изв. АН СССР. Мех. твердого тела. 1973. № 4. С. 7-13.

[6] Федосенко Ю.С. О структуре фазового пространства и периодических движениях неавтономных динамических систем с ударными взаимодействиями // ПММ. 1976. Т. 40. № 4. C. $618-629$.

[7] Горбиков С. П. Особенности строения фазового пространства динамических систем с ударными взаимодействиями // Изв. АН СССР. Мех. твердого тела. 1987. № 3. С. 23-26.

[8] Горбиков С. П. Локальные особенности динамических систем с ударными взаимодействиями // Матем. заметки. 1998. Т. 64. № 4. С. 531-542.

[9] Горбиков С. П. Дифференциальные уравнения, определяемые динамическими системами с ударными взаимодействиями на гиперповерхности удара // Вестн. Нижегородского гос. ун-та. Матем. моделирование и оптимальное управление. Вып. 1(18). Н. Новгород: Изд-во Нижегородского ун-та, 1998. С. 43-52.

[10] Горбиков С. П. Структура фазового пространства динамических систем с ударньми взаимодействиями. Деп. в ВИНИТИ СССР 23.05.85 № 3559. Горький: Горьк. гос. ун-т, 1985. $15 \mathrm{c.}$.

[11] Неймарк Ю. И. Метод точечных отображений в теории нелинейных колебаний. М., 1972.

[12] Фейгин М.И. Скользящий режим в динамических системах с ударньми взаимодействиями // ПММ. 1967. Т. 31. № 3. С. 533-536.

[13] Нагаев Р. Ф. Механические процессы с повторными затухающими соударениями. М.: Наука, 1985.

[14] Мищенко А. С., Фоменко А. Т. Курс дифференциальной геометрии и топологии. М.: Изд-во Московского ун-та, 1980.

НИИ прикладной математики и кибернетики

Поступило при Нижегородском государственном университете

03.12 .1999

им. Н. И. Лобачевского

Исправленный вариант

30.11 .2000 